\title{
Modelagem matemática e análise da hidratação de grãos de ervilha
}

\author{
Mathematical modeling and analysis of pea grains hydration
}

\author{
Edilson Sadayuki OMOTO${ }^{1}$, Cid Marcos Gonçalves ANDRADE ${ }^{1}$, Regina Maria Matos JORGE ${ }^{1}$, \\ Mônica Ronobo COUTINHO ${ }^{1}$, Paulo Roberto PARAÍSO ${ }^{1}$, Luiz Mario de Matos JORGE ${ }^{1 *}$
}

\section{Resumo}

Desenvolveu-se um modelo matemático para estimar a concentração de água em grãos de ervilha durante sua hidratação, o qual foi obtido a partir de um balanço de massa em regime transiente, considerando-se volume constante e geometria esférica. Este modelo é representado por uma equação diferencial ordinária de primeira ordem e possui dois parâmetros: o coeficiente de transferência de massa $\left(K_{s}\right)$ e a concentração de equilíbrio $\left(\rho_{\text {Aeq }}\right)$. Ambos foram ajustados numericamente tomando-se como critério a minimização da soma dos resíduos quadráticos, utilizando-se dados experimentais de hidratação de grãos de ervilha a 20, 30, 40, 50 e $60^{\circ} \mathrm{C}$. Em todas as temperaturas, o modelo representou as principais tendências do processo de hidratação com desvios inferiores a $5 \%$. Foi observado que $\mathrm{K}_{s}$ apresentou um comportamento segundo a equação de Arrhenius frente à temperatura, $K_{s}=182,8 \exp (-3521 / T)$, enquanto $\rho_{\text {Aeq }}$ permaneceu praticamente constante, obtendo-se um valor médio de 0,53 g.mL $\mathrm{mL}^{-1}$. Utilizando-se a correlação de $\mathrm{K}_{\mathrm{s}}$ e assumindo-se o valor de $\rho_{\text {Aeq }}$ médio, obteve-se um modelo generalizado, $\mathrm{o}$ qual apresentou para todos os dados um desvio ligeiramente superior a 7\%. Adicionalmente, constatou-se que a densidade dos grãos de ervilha permaneceu praticamente constante ao longo da hidratação independentemente da temperatura.

Palavras-chave: temperatura de hidratação; concentração de água; equilíbrio; coeficiente de transferência de massa.

\begin{abstract}
A mathematical model for estimating the water concentration in pea grains during hydration was developed from a transient water mass balance inside a grain of pea considering a constant volume and spherical geometry. This model is represented by an ordinary first order differential equation and has two parameters: the mass transfer coefficient $\left(\mathrm{K}_{\mathrm{s}}\right)$ and the equilibrium concentration $\left(\rho_{\text {Aeq }}\right)$. Both parameters have been numerically fitted using the minimization of the sum-of-squared residuals criterion and the hydration experimental data for pea grains at $20,30,40,50$, and $60^{\circ} \mathrm{C}$. At all temperatures, the model represented the major trends of the hydration process with standard deviations smaller than 5\%. For the model fitted parameters, it was observed that the mass transfer coefficient behaved according to Arrhenius's equation in relation to the temperature variation, $\mathrm{K}_{\mathrm{s}}=182.8 \cdot \exp (-3521 / \mathrm{T})$ while the equilibrium concentration remained practically constant showing an average value of $\rho_{\text {Aeq }}=0.533 \mathrm{~g} / \mathrm{cm}^{3}$. Using the Ks Arrhenius's correlation and assuming the average value of $\rho_{\text {Aeq }}$, a generalized model was obtained, which resulted in a standard deviation slightly greater than $7 \%$ for all the data values. Additionally, it was observed that the pea grains density remains practically constant along the hydration process and does not depend on the temperature.
\end{abstract}

Keywords: hydration temperature; water content; equilibrium; mass transfer coefficient.

\section{Introdução}

A ervilha (Pisum sativum) é um alimento muito consumido na forma fresca ou na industrializada como alimento enlatado. Sua constituição química é de 6,7\% de proteína, $0,5 \%$ de óleo e $13,9 \%$ carboidratos, sendo uma importante fonte de fibras e proteínas (SEHIRALI, 1998).

O processamento da ervilha requer grãos com propriedades físicas bem definidas, o que está diretamente relacionado com o seu teor de umidade inicial (YALÇIN, 2007), necessitando, na maioria das vezes, de um pré-tratamento dos grãos antes de serem processados, sendo, o mais comum, a hidratação dos grãos, a qual pode ser realizada de diversas maneiras, destacando-se: a) imersão de grãos em água (BECKER; SALLANS, 1955); b) exposição dos grãos a uma atmosfera de vapor saturado (AGUERRE; SUAREZ; VIOLLAZ, 1982; NOVAIS, 1990); e c) percolação de um leito de grãos por ar úmido (MANTOVANI, 1976).
O processo de hidratação de grãos também está presente na caracterização da qualidade fisiológica do grão (ROCHA et al.,1984; SOUZA; JULIO FILHO; NOGUEIRA, 1996), na extração de algum constituinte de interesse, no cozimento (WILLIANS; NAKOUL; SINGH, 1983; DESPANDE; CHERYAN, 1986; THANOS, 1998), na redução ou eliminação de fatores antinutricionais existentes nos grãos (KHOKAR; CHANHAN, 1986a) e na melhora da digestibilidade (GEERNANI; THEOPHILUS, 1980; KHOKAR; CHANHAN, 1986b). Em todos estes casos há a necessidade de grãos com teores de umidade específicos.

A absorção de água pela ervilha depende de vários fatores, dentre os quais, podem ser destacados: a composição química dos grãos, a permeabilidade, a disponibilidade de água nos estados líquido e gasoso (MAYER; MAYBER, 1978), a temperatura

Recebido para publicação em 23/3/2007

Aceito para publicação em 3/1/2009 (002416)

Programa de Pós-Graduação em Engenharia Química - PEQ, Universidade Estadual de Maringá, Av. Colombo, 5790, Bloco E46, Sala 9, CEP 87020-900, Maringá - PR, Brasil,E-mail:lmmj@deq.uem.br

${ }^{*}$ A quem a correspondência deve ser enviada 
de hidratação (WAGGONER; PARLANGE, 1976; MURPHY; NOLAND, 1982), a área de contato entre o grão e o substrato (CALERO; WEST; HINSON, 1981; SHIEH; MCDONALD, 1982; LEDEUNF, 1989) e o teor inicial de água das sementes (HOBBS; OBENDORF, 1972; VERTUCCI; LEOPOLD, 1987). Uma vez que a ervilha pode ser utilizada em diferentes graus de hidratação, há um grande interesse em caracterizar a dinâmica deste processo a fim de encontrar as melhores condições de temperatura e de tempo de embebição para alcançar o teor de umidade desejado. Para isso, é necessário desenvolver um modelo que contemple as principais características do processo. Os modelos matemáticos podem ser divididos em dois grupos principais: empíricos e fenomenológicos.

Os modelos empíricos geralmente são obtidos a partir de simples correlações matemáticas dos dados experimentais (SINGH; KULSHRESTHA, 1987; PELEG, 1988; PAN; TANGRATANAVALEE, 2003) e os seus parâmetros, normalmente, não possuem significado físico. Por sua vez, os modelos fenomenológicos consideram as etapas elementares de transferência de massa (HSU, 1983a,b; COUTINHO et al., 2005) e os seus parâmetros, frequentemente, apresentam significado físico. Dentre os parâmetros com significado físico frequentemente envolvidos, podem-se citar a concentração de equilíbrio $\left(\rho_{\text {Aeq }}\right)$ e o coeficiente de transferência de massa por convecção $\left(K_{s}\right)$.

Neste contexto, pretende-se desenvolver um modelo fenomenológico de parâmetros concentrados e validá-lo frente às medidas de concentração de água nos grãos de ervilha efetuadas em laboratório ao longo da hidratação por imersão em água a várias temperaturas. Adicionalmente, deseja-se avaliar a influência da temperatura sobre o tempo de hidratação, bem como sobre os parâmetros do modelo.

\section{Material e métodos}

\subsection{Equipamento utilizado}

O equipamento utilizado neste trabalho consiste de um banho termostático com controle de temperatura, Modelo MA184, conforme ilustrado na Figura 1, dentro do qual foi colocada uma fôrma retangular de alumínio de $30 \mathrm{~cm}$ de comprimento, $18 \mathrm{~cm}$ de largura e $5 \mathrm{~cm}$ de altura. No interior da fôrma, encontravamse dispostos água e grãos de ervilha.

\subsection{Procedimento experimental}

Os experimentos foram realizados adequando-se a metodologia apresentada por Coutinho et al. (2005), conforme sequência detalhada abaixo:

1) Regulou-se o banho termostático para operar a 20,30, 40, 50 e $60^{\circ} \mathrm{C}$ e aguardou-se até que este alcançasse a condição de regime permanente;

2) Colocou-se a fôrma sobre o banho e acrescentaram-se aproximadamente $1000 \mathrm{~mL}$ de água na mesma temperatura do banho;
3) Adicionaram-se aproximadamente $200 \mathrm{~g}$ de ervilha lisa à água no interior da fôrma, cuja umidade inicial era em torno de $15 \%$ (b.u.);

4) Retirou-se uma amostra de aproximadamente $20 \mathrm{~g}$ de ervilha do recipiente, em intervalos de tempo prédeterminados: $1,5,10,20,30,50,70,90,120,150 \mathrm{e}$ 180 minutos;

5) Espalhou-se cada amostra de grãos sobre papel toalha para retirar a água superficial;

6) Utilizou-se uma parte da amostra para avaliar a densidade do grão de ervilha (por picnometria), enquanto a outra parte destinou-se à determinação da umidade em base úmida $\left(X_{b u}\right)$, de acordo com a Equação 1, medindo-se o peso da amostra de ervilhas antes $(M U)$ e depois $(M S)$ da secagem pelo método clássico de secagem em estufa a $105^{\circ} \mathrm{C}$ durante 24 horas ou até peso constante;

$X_{b U}=\frac{M U-M S}{M U}$

7) Calculou-se a concentração mássica de água na ervilha $\left(\rho_{A}\right)$ a partir da Equação 2, conhecendo-se $X_{b U}$ e a densidade dos grãos de ervilha $\left(\rho_{e r v}\right)$.

$\rho_{A}=X_{b u} \cdot \rho_{e r v}$

\subsection{Modelagem matemática}

Partindo-se de um balanço de massa em regime transiente para a água contida na ervilha e, considerando-se a concentração de água uniforme dentro do grão, obtém-se a Equação 3, a qual é a base do modelo matemático desenvolvido. Esta equação representa a variação da massa de água no grão $\left(\rho_{A} V\right)$ com o tempo $(t)$. Sendo $A$ a área externa do grão; $V$, o volume do grão; e $N_{\mathrm{A}}$ o fluxo mássico de água $\left(\mathrm{g} \cdot \mathrm{cm}^{-2} \mathrm{~min}^{-1}\right)$.

$$
\frac{d\left(\rho_{A} V\right)}{d t}=N_{A} \cdot A
$$

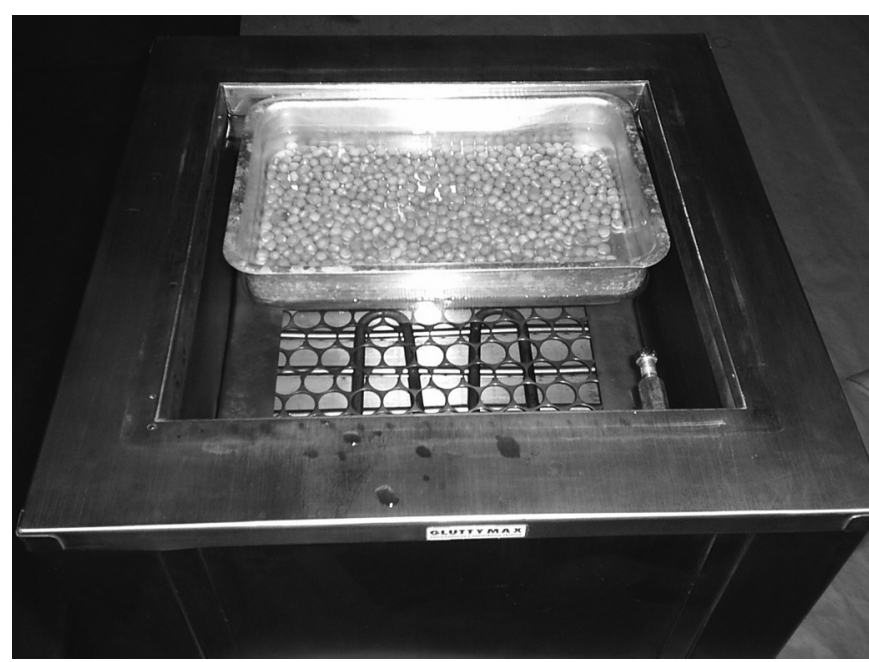

Figura 1. Banho termostático modelo MA184. 
Considerando-se o volume constante, a geometria esférica do grão com raio $r$, definindo-se o fluxo mássico como sendo $N_{A}=K_{s}\left(\rho_{e q}-\rho_{A}\right)$ e fazendo-se essas substituições na Equação 3, chega-se à forma final do modelo descrita pela Equação 4. Este modelo possui dois parâmetros: o coeficiente de transferência de massa, $K_{\mathrm{s}},\left(\mathrm{cm} \cdot \mathrm{min}^{-1}\right)$ e a concentração de água no grão no equilíbrio, $\rho_{e q}\left(\mathrm{~g} . \mathrm{cm}^{-3}\right)$ e pode ser resolvido admitindo-se como condição inicial que, no início da hidratação, $t=0$, a concentração de água no grão é uniforme e conhecida, $\rho_{A}=\rho_{\text {A o. }}$.

$\frac{d\left(\rho_{A}\right)}{d t}=\frac{3 K s}{r_{0}}\left(\rho_{A e q}-\rho_{A}\right)$

\subsection{Solução numérica e ajuste dos parâmetros do modelo}

Tanto a solução numérica como o ajuste dos parâmetros do modelo $\left(K_{s}\right.$ e $\left.\rho_{e q}\right)$ foram efetuados no MATLAB e estão representados no fluxograma contido na Figura 2. O modelo (Equação 4) foi resolvido numericamente utilizando-se a rotina ode45, enquanto o ajuste dos dois parâmetros $\left(\mathrm{K}_{\mathrm{s}}\right.$ e $\left.\rho_{\mathrm{eq}}\right)$ foi efetuado a partir da minimização da função objetivo $\left(\phi^{2}\right)$ definida pela Equação 5 , utilizando-se a rotina fminsearch. Esta função objetivo representa a somatória das diferenças entre as concentrações de água nos grãos de ervilha medidas experimentalmente ao longo da hidratação, $\rho_{\text {A.exp }}$, em $n$ instantes de tempo e as concentrações da água na ervilha calculadas pelo modelo, $\rho_{\text {Acal }}$, para uma dada temperatura: $20,30,40,50$ e $60^{\circ} \mathrm{C}$.

$\varphi^{2}=\sum_{1}^{n}\left(\rho_{A c a l}^{i}-\rho_{A \exp }^{i}\right)^{2}$

\section{Resultados e discussão}

\subsection{Comportamento de $\rho_{\text {erv }}$ ao longo da hidratação}

Conforme se pode constatar pela análise da Figura 3, ao longo da hidratação, o teor de umidade em base úmida $\left(\mathrm{X}_{\mathrm{bu}}\right)$ vai

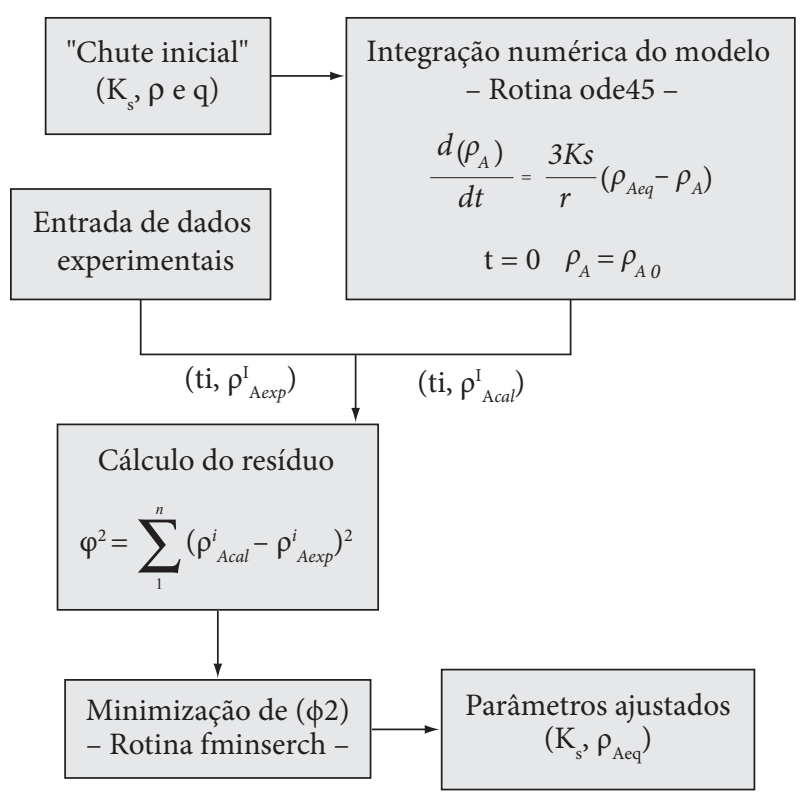

Figura 2. Esquema da técnica de ajuste utilizada. aumentando, entretanto, a densidade do grão $\left(\rho_{e v v}\right)$ permanece praticamente constante, com um valor médio de $1 \mathrm{~g} / \mathrm{cm}^{3}$. Este resultado concorda com o trabalho de Yalçin, Ozarslan e Akbas (2006) e sugere que a densidade dos sólidos constituintes da ervilha deve ser próxima à da água.

\subsection{Simulação e ajuste dos parâmetros do modelo}

Os valores dos parâmetros do modelo, ajustados individualmente para cada temperatura de hidratação, juntamente com o seu desvio quadrático acumulado $\left(\phi^{2}\right)$, estão apresentados na Tabela 1.

Os resultados das simulações do modelo frente aos dados experimentais estão apresentados na Figura 4, indicando que tanto o modelo proposto como as técnicas utilizadas na solução numérica e o ajuste dos parâmetros são adequados.

Inspecionando-se a Figura 5, pode-se obter uma visão global dos desvios do modelo frente aos dados experimentais para todas as temperaturas de hidratação. Constata-se que o modelo apresentou um bom ajuste, sendo capaz de descrever o processo de hidratação com um desvio máximo de $\pm 5 \%$.

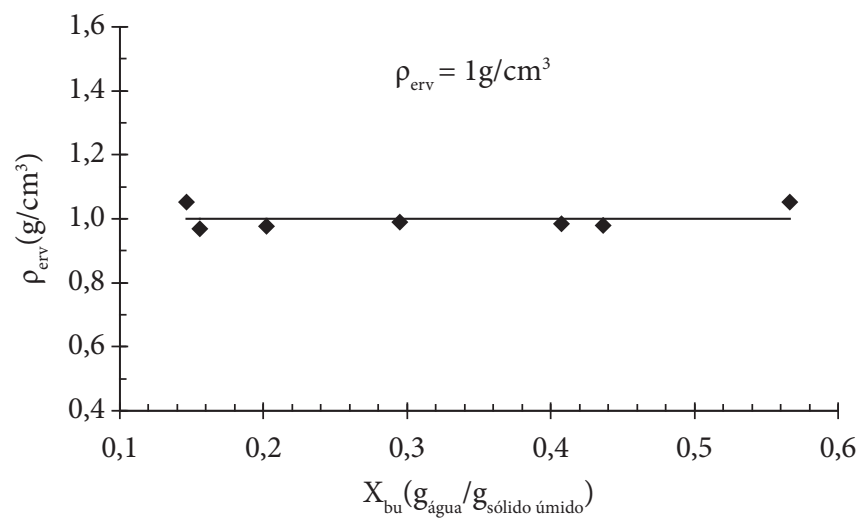

Figura 3. Influência da umidade sobre a densidade do grão.

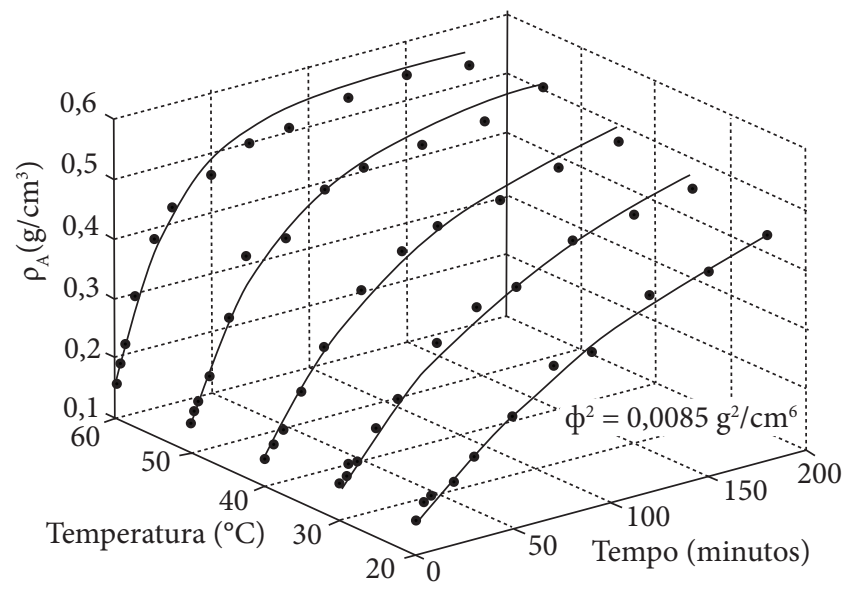

Figura 4. Simulação do modelo frente a dados experimentais. 
Tabela 1. Parâmetros ajustados do modelo.

\begin{tabular}{ccccc}
\hline $\begin{array}{c}\mathrm{T} \\
\left({ }^{\circ} \mathrm{C}\right)\end{array}$ & $\begin{array}{c}\mathrm{K}_{\mathrm{s}} \times 10^{3} \\
(\mathrm{~cm} / \mathrm{min})\end{array}$ & $\begin{array}{c}\rho \mathrm{A}_{\mathrm{eq}} \\
\left(\mathrm{g} / \mathrm{cm}^{3}\right)\end{array}$ & $\begin{array}{c}\phi^{2} \times 10^{3} \\
\left(\mathrm{~g}^{2} / \mathrm{cm}^{6}\right)\end{array}$ & $\mathrm{n}$ \\
\hline 20 & 1,003 & 0,544 & 1,40 & 11 \\
30 & 1,260 & 0,522 & 1,20 & 12 \\
40 & 1,645 & 0,535 & 1,50 & 11 \\
50 & 2,578 & 0,537 & 2,90 & 12 \\
60 & 4,270 & 0,526 & 1,50 & 12 \\
\hline
\end{tabular}

\subsection{Influência da temperatura sobre os parâmetros do modelo}

A partir da análise da Tabela 1 , pode-se constatar que $\mathrm{K}_{\mathrm{s}}$ apresenta um comportamento crescente em função da temperatura, T, o qual pôde ser bem correlacionado a partir do ajuste de uma equação matemática similar à de Arrhenius, Equação 6.

$$
K_{s}=B \cdot \exp \left(-\frac{E^{\prime}}{T}\right)
$$

Os parâmetros B, e E’ foram ajustados por simples regressão linear da Equação 7, que corresponde à forma linearizada da Equação 6, obtendo-se assim valores de B e E' correspondentes a $182,80(\mathrm{~cm} / \mathrm{min})$ e $3521\left(\mathrm{~K}^{-1}\right)$, respectivamente, com um coeficiente de correlação de 0,96 , conforme apresentado na Figura 6.

$$
\ln \left(K_{s}\right)=\ln (B)-E^{\prime} \cdot \frac{1}{T}
$$

Na Figura 7, pode-se observar tanto o comportamento de $K_{s}$ em função da temperatura como o comportamento da Equação 6 com os parâmetros ajustados, reafirmando que o modelo utilizado para representar $K_{s}$ em função da temperatura é adequado.

Além da temperatura, o tipo de grão também exerce influência significativa sobre $K_{s}$. Conforme se pode observar na Tabela 2 , os valores $K_{s}$ obtidos por Coutinho (2006) para a hidratação de grãos de soja são significativamente superiores àqueles obtidos neste trabalho com grãos de ervilha. Tanto o valor de $K_{s}$ analisado individualmente para cada grão como a diferença entre os valores de $K_{s}$ da soja e da ervilha aumentam com a elevação da temperatura.

Apesar da importância do conhecimento do $K_{s}$ para o projeto, simulação, otimização e análise de processos envolvendo a hidratação de grãos, há poucos trabalhos apresentados em literatura envolvendo a determinação desta propriedade de transporte, apontando para a necessidade de ampliar os estudos sobre este assunto.

Conforme se pode observar pela inspeção da Figura 8, a temperatura não exerceu nenhuma influência significativa sobre a concentração de equilíbrio, $\rho_{\text {Aeq }}$. Desta forma, admitiu-se um valor constante para este parâmetro igual a $0,53 \mathrm{~g} / \mathrm{cm}^{3}$, o qual foi obtido a partir da média aritmética dos valores encontrados, Tabela 1, na faixa das temperaturas exploradas.

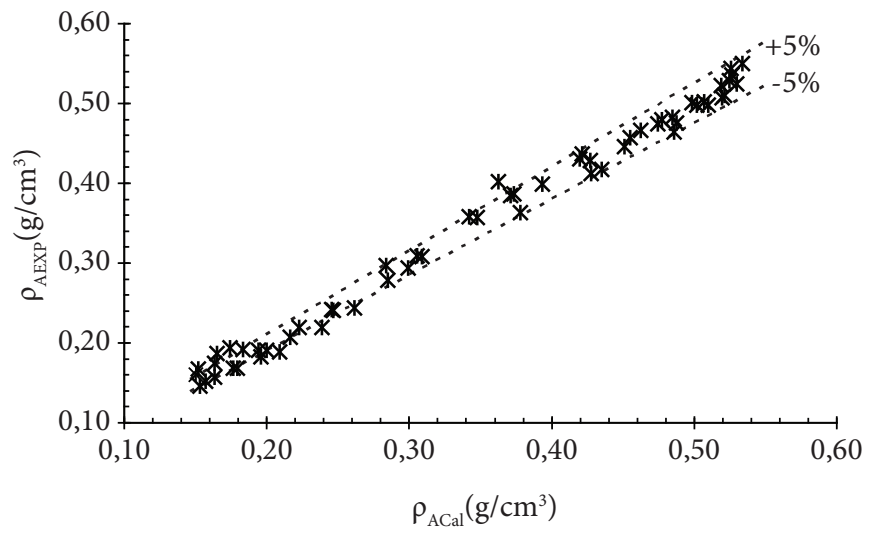

Figura 5. Desvios do modelo frente aos dados experimentais.

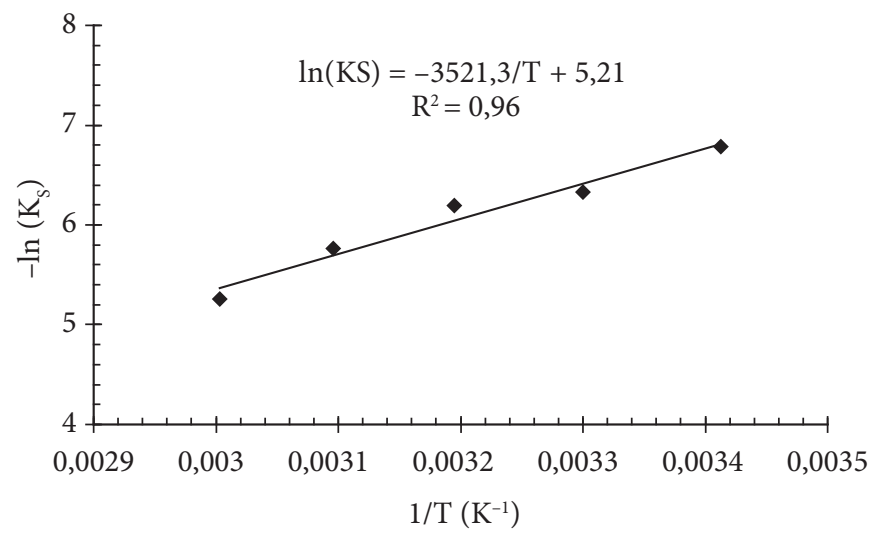

Figura 6. Qualidade de ajuste da Equação 7.

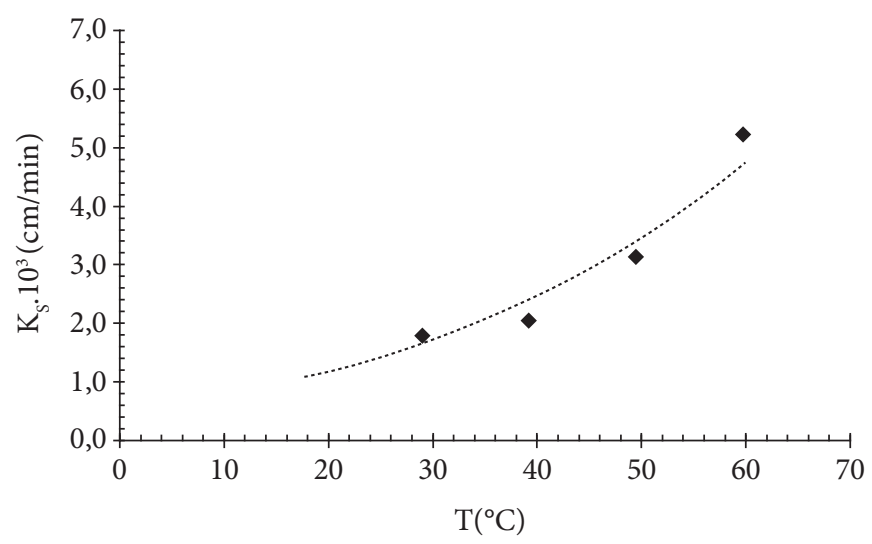

Figura 7. Influência da temperatura sobre $K_{s}$.

A qualidade da estimativa para o valor médio da umidade de equilíbrio $\left(0,53 \mathrm{~g} / \mathrm{cm}^{3}\right)$ pôde ser comprovada efetuando-se a comparação destes resultados com os de literatura. Krokida e Marinos-kouris (2003) efetuaram diversos ensaios experimentais de hidratação de grãos de ervilha a 40 e $60^{\circ} \mathrm{C}$, conduzindo a valores de $\rho_{\text {Aeq }}$ de 0,54 e $0,58 \mathrm{~g} / \mathrm{cm}^{3}$, respectivamente, cujo valor médio é de $0,56 \mathrm{~g} / \mathrm{cm}^{3}$, o qual é apenas $5,4 \%$ superior ao valor médio obtido neste trabalho. 
Tabela 2. Influência do tipo de grão sobre $\mathrm{K}_{\mathrm{s}}$.

\begin{tabular}{cccc}
\hline & Ervilha & Soja & \\
\hline $\begin{array}{c}\mathrm{T} \\
\left({ }^{\circ} \mathrm{C}\right)\end{array}$ & $\begin{array}{c}\mathrm{K}_{\mathrm{s}} \times 10^{3} \\
(\mathrm{~cm} / \mathrm{min})\end{array}$ & $\begin{array}{c}\mathrm{K}_{\mathrm{s}} \times 10^{3} \\
(\mathrm{~cm} / \mathrm{min})\end{array}$ & $\begin{array}{c}\text { Diferença } \times 10^{3} \\
(\mathrm{~cm} / \mathrm{min})\end{array}$ \\
\hline 20 & 1,11 & 9,60 & 8,49 \\
30 & 1,79 & 11,70 & 9,91 \\
50 & 3,14 & 13,90 & 10,76 \\
\hline
\end{tabular}

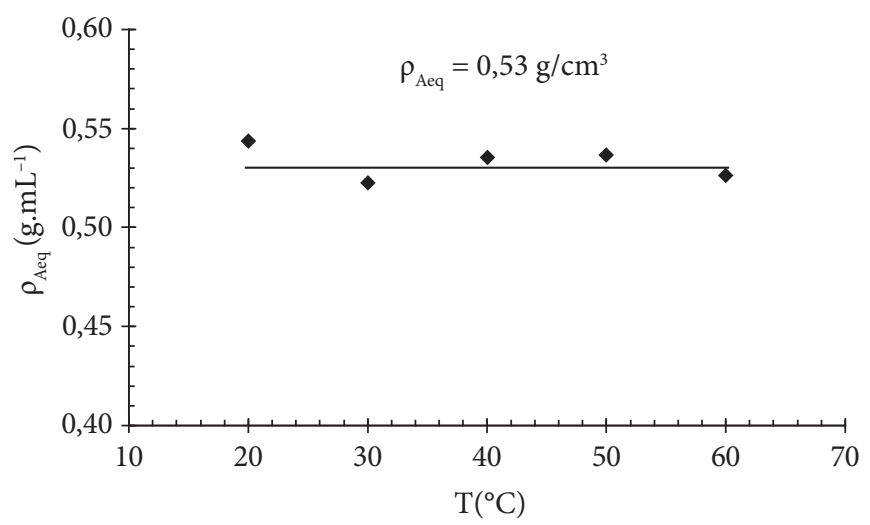

Figura 8. Influência da temperatura sobre $\rho A_{e q}$.

\subsection{Influência da temperatura sobre $t_{e q}$}

Considerando-se que tanto $\mathrm{\rho A}_{\mathrm{eq}}$ como $\mathrm{K}_{\mathrm{s}}$ sejam constantes para uma dada temperatura de hidratação, pode-se integrar a Equação 4, obtendo-se a Equação 8.

$\ln \left(\frac{\rho_{A e q}-\rho_{A}}{\rho_{A e q}-\rho_{A O}}\right)=-\frac{3 K_{s}}{r_{o}} t$

Definindo-se o tempo para os grãos de ervilha alcançarem a condição de equilíbrio $\left(t_{e q}\right)$ como sendo aquele para o qual a umidade em que os grãos atingem $99 \%$ da umidade de equilíbrio $\left(\rho_{A}=0,99 \rho_{\text {Aeq }}\right)$, pode-se reescrever a Equação 8 na forma da Equação 9.

$t_{e q}=\frac{r_{o}}{3 K_{S}} \ln \left(\frac{100\left(\rho_{A e q}-\rho_{A o}\right)}{\rho_{\text {Aeq }}}\right)$

Na Figura 9, pode-se observar o comportamento de $t_{e q} \mathrm{em}$ função da temperatura, obtido a partir da Equação 7, admitindose $\rho_{\text {Aeq }}=0,53$ e $K_{s}=182,80 \exp (-3521 / T)$. Observa-se que o aumento da temperatura provoca uma queda exponencial no tempo de equilíbrio, o qual varia de 450 a 150 minutos para temperaturas entre 20 e $60^{\circ} \mathrm{C}$.

Klamczynska, Czuchajowska e Baik (2001) efetuaram prolongados ensaios de hidratação a temperatura ambiente $\mathrm{e}$ constataram que as ervilhas lisas demoram, no mínimo, 8 horas para alcançarem as condições de equilíbrio. Admitindo-se a temperatura ambiente de $20^{\circ} \mathrm{C}$ na Figura 9 , encontra-se o valor de 7,5 horas para $t_{e q}$, o qual é 6,5\% inferior ao valor apontado pelos autores acima, reafirmando a validade da Equação 9.

\subsection{Modelo generalizado para hidratação}

O modelo generalizado corresponde à Equação 4 utilizando-se a correlação de $\mathrm{K}_{\mathrm{s}}$ em função da temperatura (Equação 6) e admitindo-se um valor médio para $\rho_{\text {Aeq }}\left(0,53 \mathrm{~g} \cdot \mathrm{mL}^{-1}\right)$. A simulação deste modelo é capaz de estimar a concentração de água na ervilha em diferentes condições de temperatura e de tempo da hidratação. A Figura 10 ilustra os resultados das simulações do modelo generalizado em função do tempo e da temperatura, sendo que a superfície esboçada na figura representa o campo de simulação do modelo, enquanto os pontos discretos correspondem aos valores experimentais obtidos neste trabalho. Observa-se que o modelo generalizado representou satisfatoriamente as principais tendências do processo de hidratação, apresentando um resíduo quadrático acumulado $\left(\phi^{2}\right)$ para todas as temperaturas de $0,0119 \mathrm{~g}^{2} / \mathrm{cm}^{6}$.

A qualidade das previsões do modelo generalizado também pode ser observada na Figura 11, revelando que os desvios do modelo generalizado, frente a todos os dados experimentais, foram de, no máximo, 7\%. Este valor é um pouco superior àquele obtido a partir dos ajustes individuais do modelo (5\%),

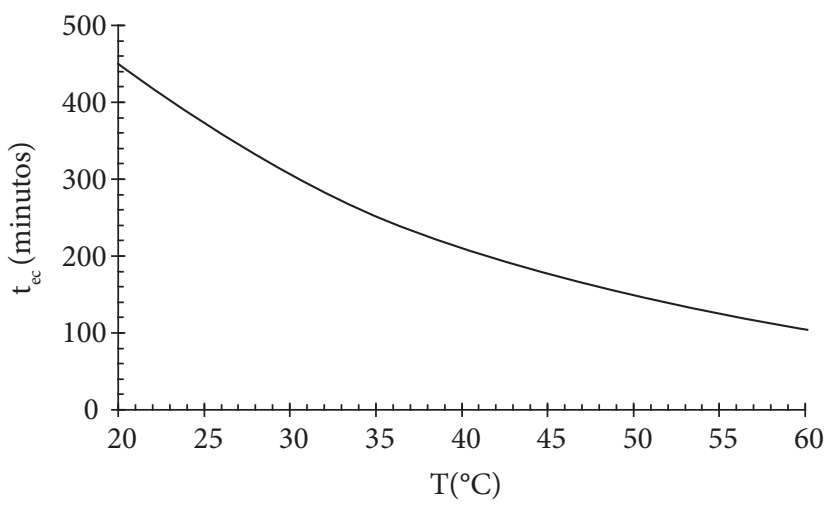

Figura 9. Tempo de equilíbrio em função da temperatura.

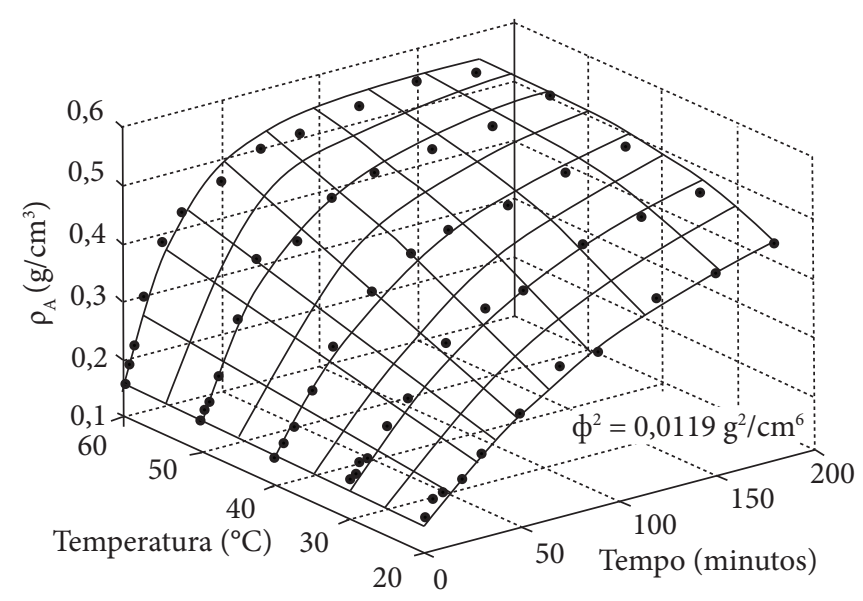

Figura 10. Simulação do modelo generalizado frente a dados experimentais. 


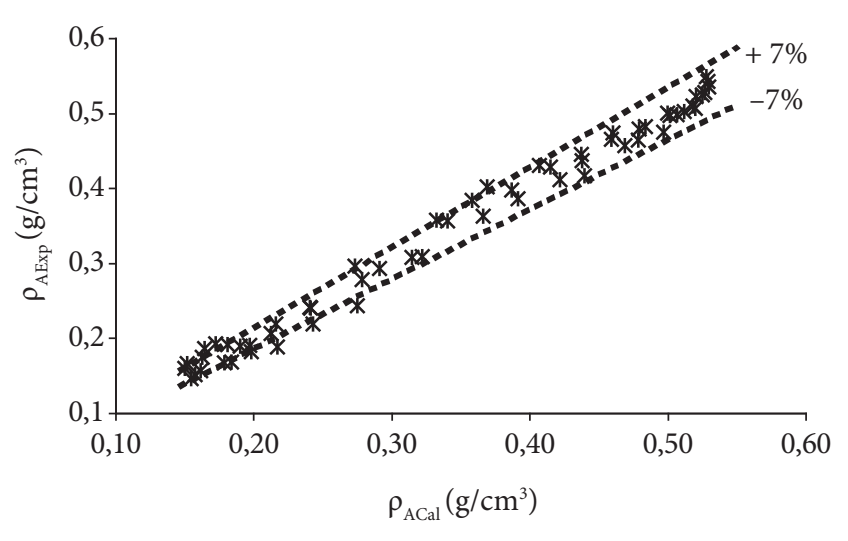

Figura 11. Esquematização dos desvios do modelo generalizado.

justificando a utilização do modelo generalizado como ferramenta preliminar para a estimativa de tempos de hidratação necessários para alcançar um certo teor de umidade numa dada temperatura, na faixa de condições experimentais explorada.

\section{Conclusões}

A densidade dos grãos de ervilha é praticamente constante ao longo da hidratação e possui um valor médio de $1 \mathrm{~g} / \mathrm{cm}^{3}$.

O processo de hidratação foi representado satisfatoriamente pelo modelo matemático desenvolvido, apresentando um desvio máximo de $5 \%$.

Os valores do coeficiente de transferência de massa por convecção, $K_{s}$, variaram entre $1 \times 10^{-3}$ e $6 \times 10^{-3} \mathrm{~cm} \cdot \mathrm{min}^{-1}$ na faixa de temperaturas de hidratação entre 20 e $60^{\circ} \mathrm{C}$.

$\mathrm{O}$ parâmetro $\mathrm{K}_{\mathrm{s}}$ pode ser correlacionado com a temperatura a partir do ajuste de uma equação similar à de Arrhenius, $K_{\mathrm{s}}=B \cdot \exp \left(-E^{\prime} / T\right)$, obtendo-se $\mathrm{B}=182,8 \mathrm{~cm} \cdot \mathrm{min}^{-1} \mathrm{e} \mathrm{E}^{\prime}=3521 \mathrm{~K}^{-1}$ com um coeficiente de correlação de 0,96 .

A temperatura não exerce influência significativa sobre a concentração de equilíbrio da água nos grãos de ervilha, $\rho_{\text {Aeq' }}$ na faixa de temperaturas explorada, obtendo-se um valor médio de $0,53 \mathrm{~g} / \mathrm{cm}^{3}$.

O tempo no qual os grãos de ervilha atingem a condição de equilíbrio, $t_{e q}$, decai acentuadamente com o aumento da temperatura, variando entre 450 e 150 minutos na faixa de temperaturas de 20 e $60^{\circ} \mathrm{C}$, respectivamente.

$\mathrm{O}$ modelo generalizado representou satisfatoriamente as principais tendências do processo de hidratação com um desvio máximo de 7\%, podendo ser utilizado como ferramenta preliminar para a estimativa de tempos de hidratação necessários para alcançar um certo teor de umidade numa dada temperatura, na faixa de condições experimentais explorada, sem a necessidade de ajuste de nenhum parâmetro.

\section{Referências bibliográficas}

AGUERRE, R.; SUAREZ, C.; VIOLLAZ, P. E. Drying kinetics of rough rice grain. Journal of Food Technology, v. 17, n. 6, p. 679-686, 1982.
BECKER, H. A.; SALLANS, H. R. A study of diffusion in solids of arbitrary shape, with application to the drying of the wheat kernel. Cereal Chemistry, v. 32, p. 212-226, 1955.

CALERO, E.; WEST, S. H.; HINSON, K. Water absortion of soybean seeds and associated causal factors. Crop Science, v. 21, n. 6, p. 926-933, 1981.

COUTINHO, M. R. et al. Modelagem e validação da hidratação de grãos de soja. Ciência e Tecnologia de Alimentos, v. 25, n. 3, p. $603-610,2005$.

COUTINHO, M. R. Modelagem, simulação e análise da hidratação de grãos de soja. Maringá, PR, 2006. 178 p. Tese (Doutorado em Engenharia Química) - Departamento de Engenharia Química, Universidade Estadual de Maringá - UEM.

DESPANDE, S. S.; CHERYAN, M. Water uptake during cooking of dry beans. Plant Foods for Human Nutrition, v. 36, n. 3, p. $157-165,1986$.

GEERNANI, P. E.; THEOPHILUS, F. Effect of home processing on the protein quality of selected legumes. Journal of Food Science, v. 45, n. 3, p. 707-709, 1980.

HOBBS, P. R.; OBENDORF, R. L. Interaction of initial seed moisture and imbibitional temperature on germination and productivity of soybean. Crop science, v. 13, n. 5, p. 664-667, 1972.

HSU, K. H. A diffusion model with a concentration-dependent diffusion coefficient for describing water movement in legumes during soaking. Journal of Food Science, v. 48, n. 2, p. 618-622, 1983.

HSU, K. H. Effect of temperature on water diffusion in soybean. Journal of Food Science, v. 48, n. 4, p. 1364-1365, 1983.

KHOKAR, S.; CHANHAN, B. M. Antinutricional factors in north beans (Vigna aconitifolia). Varietal differences and effects of methods of domestic processing and cooking. Journal of Food Science, v. 51, n. 3, p. 591-594, 1986a.

KHOKAR, S.; CHANHAN, B. M. Effect of domestic processing and cooking in vitro protein digestibility of north bean. Journal of Food Science, v. 51, n. 4, p. 1083-1085, 1986 b.

KLAMCZYNSKA, B.; CZUCHAJOWSKA, Z. E.; BAIK, B. Composition, soaking, cooking properties and thermal characteristics of starch of chickpeas, wrinkled peas and smooth peas. International Journal of Food Science and Technology, v. 36, n. 5, p. 563-572, 2001.

KROKIDA, M. K.; MARINOS-KOURIS, D. Rehydration kinetics of dehydrated products. Journal of Food Engineering, v. 57, n. 1, p. 1-7, 2003.

LEDEUNF, Y. Hydration des semences de pois (Pisun sativum 1.). Seed Science and Technology, v. 17, n. 3, p. 471-483, 1989.

MANTOVANI, B. H. M. Análise e Simulação de Secagem de Grãos de Milho em Camadas Espessa. Viçosa, MG, 1976. 132p. Dissertação (Mestrado em Engenharia Agrícola) - Departamento de Engenharia Agrícola, Universidade Federal de Viçosa.

MAYER, A. M.; MAYBER, A. P. The germination of seed. 2 ed. Oxford: Pergamon Press, 1978. 192 p.

MURPHY, J. B.; NOLAND, T. L. Temperature effects on seed imbibition and leakage mediated by viscosity and membranes. Plant physiology, v. 69, n. 1/3, p. 428-431, 1982.

NOVAIS, A. F. Análise da aplicação do leito deslizante e escoamentos cruzados na secagem de sementes. São Carlos, SP, 1990. 95p. Dissertação (Mestrado em Engenharia Química) - Departamento de Engenharia Química, Universidade Federal de São Carlos. 
PAN, Z.; TANGRATANAVALEE, W. Characteristics of soybean as affected by soaking conditions. Lebensmittel-Wissenschaft und -Technologie, v. 36, n. 1, p. 143-151, 2003.

PELEG, $M$. An empirical model for the description of moisture sorption curves. Journal of Food Science, v. 53, n. 4, p. 1216-1219, 1988.

ROCHA, V. S. et al. Embebição de água e qualidade fisiológica de sementes de soja. Revista Brasileira de Sementes, v. 6, n. 2, p. 51-66, 1984.

SEHIRALI, S. Yemeklik Dane Baklagiller. Ankara, Turkey: Ankara University Press, 1988.

SHIEH, W. J.; MCDONALD, M. B. The influence of seed size, shape and treatment on inbred seed corn quality. Seed Science and Technology, v. 10, n. 2, p. 307-313, 1982.

SINGH, B. P. N.; KULSHRESTHA, S. P. Kinetcs of water sorption by soybean and pigeonpea grains. Journal of Food Science, v. 52, n. 6 , p. 1538-1544, 1987.

SOUZA, F. H. D.; JUliO FILHO, M.; NOGUEIRA, M. C. S. Características físicas das sementes de calopogonium mucunoides desv. associadas à qualidade fisiológica e ao padrão de absorção de água. I. Tamanho. Revista Brasileira de Sementes, v. 18, n. 1, p. 33-40, 1996.

THANOS, A. J. Water changes in canned dry peas and beans during heat processing. International Journal of Food Science and Technology, v. 33, p. 539-545, 1998.

VERTUCCI, C. W.; LEOPOLD, C. A. Water binding in legume seeds. Plant Physiology, v. 85, n. 1, p. 224-231,1987.

WAGGONER, P. E.; PARLANGE, J. Y. Water uptake and water diffusivity of seeds. Plant physiology, v. 57, n. 1/3, p. 153-156, 1976.

WILLIANS, P. C.; NAKOUL, H.; SINGH, K. B. Relationship between cooking time and some physical characteristics in chickpeas. Journal of Science of Food Agriculture, v. 34, n. 5, p. 492-496, 1983.

YALÇIN, I.; OZARSLAN, C.; AKBAS, T. Physical properties of pea (Pisum sativum) seed. Journal of Food engineering, v. 79, n. 2, p. 731-735, 2007. 